University of Wollongong

Research Online

Faculty of Engineering and Information

Faculty of Engineering and Information

Sciences - Papers: Part A

Sciences

$1-1-2014$

How the type of input function affects the dynamic response of conducting polymer actuators

Xingcan Xiang

University of Wollongong, xx985@uowmail.edu.au

Gursel Alici

University of Wollongong, gursel@uow.edu.au

Rahim Mutlu

University of Wollongong, rmutlu@uow.edu.au

Weihua Li

University of Wollongong, weihuali@uow.edu.au

Follow this and additional works at: https://ro.uow.edu.au/eispapers

Part of the Engineering Commons, and the Science and Technology Studies Commons

Research Online is the open access institutional repository for the University of Wollongong. For further information contact the UOW Library: research-pubs@uow.edu.au 


\title{
How the type of input function affects the dynamic response of conducting polymer actuators
}

\begin{abstract}
There has been a growing interest in smart actuators typified by conducting polymer actuators, especially in their (i) fabrication, modeling and control with minimum external data and (ii) applications in bioinspired devices, robotics and mechatronics. Their control is a challenging research problem due to the complex and nonlinear properties of these actuators, which cannot be predicted accurately. Based on an input-shaping technique, we propose a new method to improve the conducting polymer actuators' command-following ability, while minimizing their electric power consumption. We applied four input functions with smooth characteristics to a trilayer conducting polymer actuator to experimentally evaluate its command-following ability under an open-loop control strategy and a simulated feedback control strategy, and, more importantly, to quantify how the type of input function affects the dynamic response of this class of actuators. We have found that the four smooth inputs consume less electrical power than sharp inputs such as a step input with discontinuous higher-order derivatives. We also obtained an improved transient response performance from the smooth inputs, especially under the simulated feedback control strategy, which we have proposed previously [X Xiang, R Mutlu, G Alici, and W $\mathrm{Li}, 2014$ "Control of conducting polymer actuators without physical feedback: simulated feedback control approach with particle swarm optimization', Journal of Smart Materials and Structure, 23]. The idea of using a smooth input command, which results in lower power consumption and better control performance, can be extended to other smart actuators. Consuming less electrical energy or power will have a direct effect on enhancing the operational life of these actuators.
\end{abstract}

\section{Keywords}

electroactive polymer actuators, smart actuators, response characterisation

\section{Disciplines}

Engineering | Science and Technology Studies

\section{Publication Details}

Xiang, X., Alici, G., Mutlu, R. \& Li, W. (2014). How the type of input function affects the dynamic response of conducting polymer actuators. Smart Materials and Structures, 23 (10), 105008-1 - 105008-11. 


\title{
How the Type of Input Function Affects Dynamic Response of Conducting Polymer Actuators?
}

\author{
Xingcan Xiang ${ }^{1}$, Gursel Alici'1,2, Rahim Mutlu' ${ }^{1}$ and Weihua Li ${ }^{1}$ \\ ${ }^{1}$ School of Mechanical, Materials and Mechatronic Engineering \\ ${ }^{2}$ ARC Centre of Excellence for Electromaterials Science \\ University of Wollongong, NSW, 2522, Australia
}

\begin{abstract}
There has been a growing interest in smart actuators typified by conducting polymer actuators especially in their (i) fabrication, modeling and control with minimum external data and (ii) applications in bio-inspired devices, robotics and mechatronics. Their control is a challenging research problem due to the complex and nonlinear properties of these actuators which cannot be predicted accurately. Based on an input shaping technique, we propose a new method to improve the conducting polymer actuators' command following ability, while minimizing their electric power consumption. We applied four input functions with smooth characteristics to a tri-layer conducting polymer actuator to experimentally evaluate its command following ability under an open-loop control strategy and a simulated feedback control strategy, and more importantly to quantify how the type of the input functions affects the dynamic response of this class of actuators. We have found that the four smooth inputs consume less electrical power than sharp inputs such as a step input with discontinuous higher order derivatives. We also obtained an improved transient response performance from the smooth inputs, especially under the simulated feedback control strategy, which we have proposed previously [13]. The idea of using a smooth input command, which results in lower power consumption and better control performance, can be extended to other smart actuators. Consuming less electrical energy or power will have a direct effect on enhancing the operational life of these actuators.
\end{abstract}

\section{Introduction}

Conducting polymers are one of a type of electrically actuated polymeric materials known as electroactive polymers (EAPs). The actuators, which are made of conducting polymers, and hence called 'conducting polymer actuators' (CPAs), are a promising class of smart actuators suitable for many cutting-edge applications in robotics and biomedical systems. Recently, significant research efforts have been dedicated to their synthesis, modeling and control due to their useful characteristics such as being lightweight, having biocompatibility, using minimal power consumption and the ability to operate in aqueous and non-aqueous media [1-4].

Since the early reviews of CPAs [5,6] and initial investigations into their control, many researchers have proposed methods to improve their performance under position control. These methods have focused on i) establishing a feedback control system using position data provided by an external sensor or sensors and 
ii) establishing inversion-based feedforward control approaches without using externally provided feedback data [7-15]. Compared to a conducting polymer actuator, the sensor used in the first approach is extremely large, which limits the CPAs' application and brings extra complexity and cost. The latter approach does not require a bulky feedback sensor but depends strongly on an accurate mathematical model of the actuator. Nevertheless, the actuators' accurate model is impossible to obtain as these actuators have nonlinear characteristics such as creep and hysteresis, and complex electrical, chemical and mechanical parameters, which are sensitive to synthesis, operation and application conditions.

The actuator's life is another important factor to consider when choosing an actuation and control scheme. Because of its operation principle, which is based on the movement of chemical species (i.e. anions and cations) along the polymer backbone, the actuator's life depends on the electrical power applied to it. As a result, it is desirable to minimize the electrical energy applied to these actuators.

In this paper, we propose to use various input functions to drive trilayer polymer actuators, which operate in air, in order to evaluate how the continuity of the input command affects the dynamic response of the actuators. With reference to the experimental results presented in this study, one obvious effect is the minimum electrical power consumed under smooth inputs, which is expected to extend the operational life of the actuators. We have used a step input, a harmonic input, a cycloidal input, an exponential input and a ramp input to evaluate the command tracking ability of the actuators under open loop control and a simulated feedback control approach, on which we have reported recently [13]. In the simulated feedback control method, we employed a PID controller whose gains are determined by a particle swarm algorithm in order to optimize the control system performance. To the best of our knowledge, this is the first time to report on the effect of the input functions on the dynamic behavior and energy consumption of the smart actuators typified by CPAs.

After introducing the structure and working principle of the CPAs in Section I, we present the experimental setup in Section II. Section III and Section IV focus on the input shaping technique and simulated feedback control strategy, respectively. We employ the five input signals under open-loop control and evaluate their dynamic response in Section $V$. How the input functions affect the dynamic response of the CPA under the simulated feedback is presented in Section VI. Section VII provides the experimental results for the CPA's steady state response. A discussion of the results, concluding remarks and future work are presented in Section VIII.

\section{Background}

\subsection{Conducting polymer actuators}

Poplypyrrole (PPy) is one of the most popularly used EAP material for actuators because of its larger strains and low or medium stress and their ability 
to operate at low voltages [15]. The structure of the bending-type PPy polymer actuators considered in this study is depicted in Figure 1. It consists of three layers. The inner porous poly(vinylidene fluoride) (Immobilon-P, Millipore) (PVDF) layer, which also acts as electrolyte reservoir, and two outer PPy layers, which are active components of the actuators. Between the PVDF layer and PPy layer, a very thin gold layer is coated to improve the conductivity of the substrate.

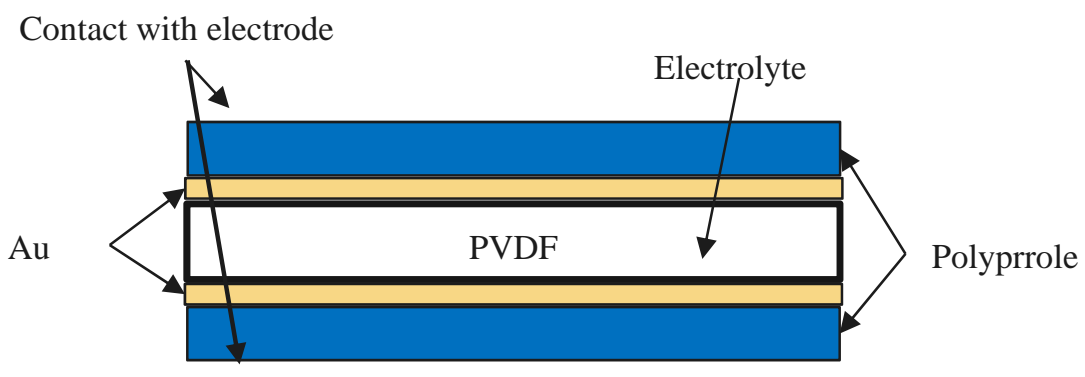

Figure 1 The structure of a PPy actuator [13]

As shown in Figure 2, when a potential difference is applied to the actuator via platinum contacts, the PPy on positive electrode is oxidized and on the other hand, the negatively charged PPy layer is reduced. The TFSI- anions in the electrolyte will move into the positively charged PPy layer and cause a volume expansion. When the TFSI- anions leave the negatively charged PPy layer, this layer will contract. As a result, the trilayer actuator will bend towards the cathode, like a one-end-fixed, the other end free cantilever beam, as shown in Figure 2.

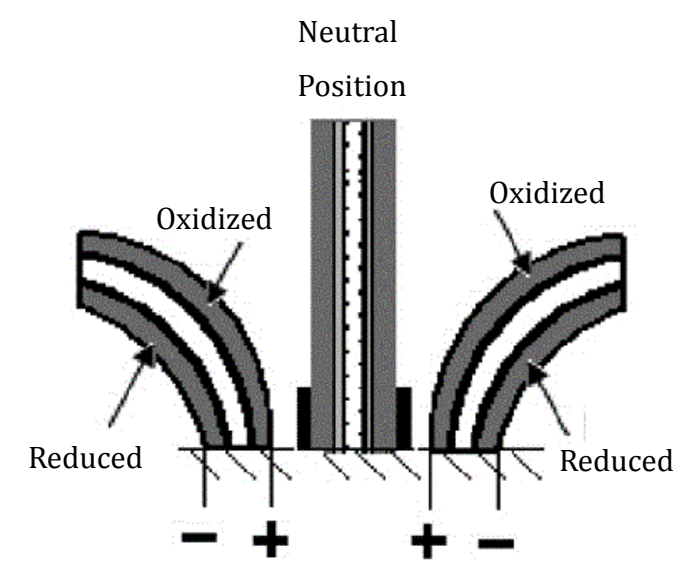

Figure 2 The bending directions of the tri-layer PPy actuator [17]. The actuator's one end is fixed to operate like a cantilever beam. While its one PPy layer is oxidized and expanding, the other is reduced and contracts, which generate a strain difference between both layers like a bimetal in order to output a displacement at its tip.

\subsection{Experimental Setup}

Figure 3 shows the experimental setup. The displacement of the CPA is measured and recorded by a laser-based displacement sensor. 


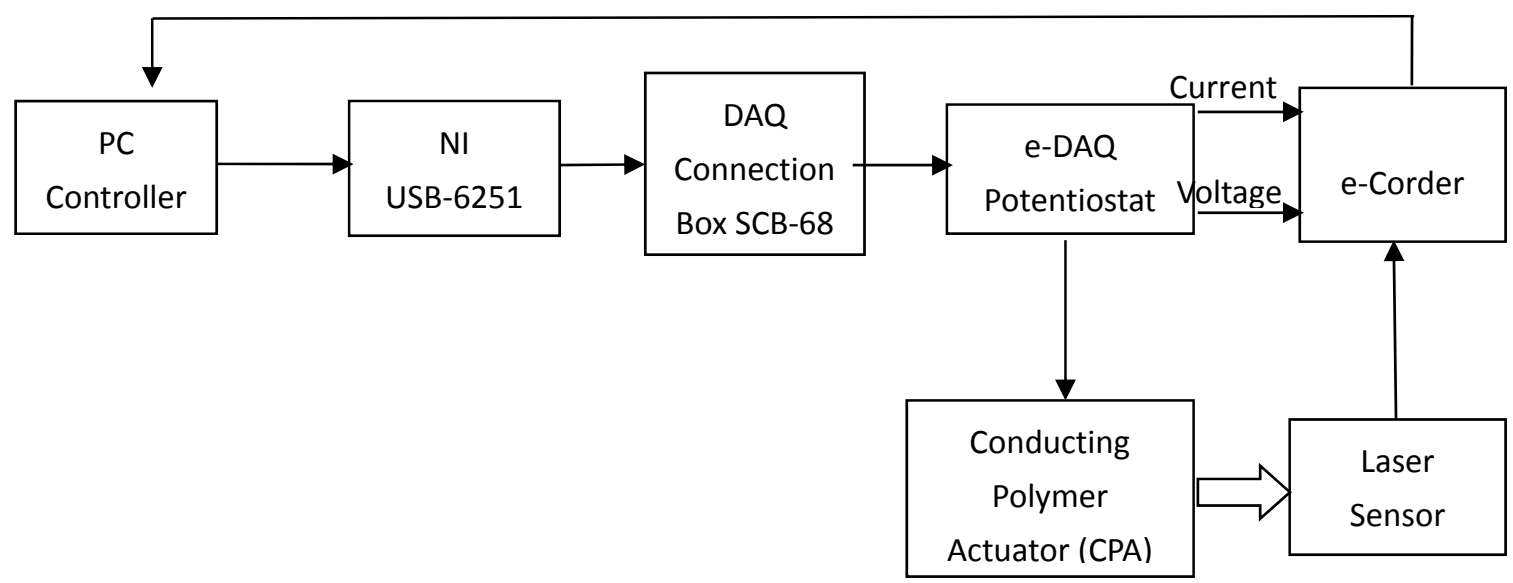

Figure 3 Schematic representation of the experimental setup [13]

\section{Input Functions}

Input shaping technique (IST) is a method to suppress residual vibrations in flexible structures. This effective and practical method has already been proven for various applications [18-21]. In this study, we evaluate the effect of the input shaping on the time-domain dynamic response of the CPAs, and the resulting electric power consumption.

The response of a CPA depends on the form of the input function. With this in mind, we aim to find a suitably shaped input that will result in minimum energy consumption, command tracking error and the best overall response performance.

A step input is a discontinuous function to command the actuator from one configuration to another. Smoother functions can be used to command the actuator to a desired configuration. The simplest of them is a ramp function, which has a constant velocity (derivative of the position input) and can be described by Eq.1. However, this function has a disadvantage of an infinite acceleration at the beginning and the end of the input. On the other hand, the harmonic and cycloidal functions described by Eq. 2 and Eq. 3, respectively, eliminate the infinite accelerations at the boundaries of the motion. We have also selected a function including exponential element described by Eq.4. This function has an infinite acceleration velocity at the beginning of the excitation but its velocity decreases over time, resulting in zero acceleration at the end of the input command. We call it exponential function in this paper.

$$
\begin{aligned}
& y_{\text {ramp }}=\frac{K_{\max }}{t_{\text {tra }}} t \\
& y_{\text {harm }}=\frac{K_{\max }}{2}\left(1-\cos \left(\pi \frac{t}{t_{\text {tra }}}\right)\right) \\
& y_{\text {cycl }}=K_{\max }\left(\frac{t}{t_{\text {tra }}}-\frac{1}{2 \pi} \sin \left(2 \pi \frac{t}{t_{\text {tra }}}\right)\right)
\end{aligned}
$$




$$
y_{\operatorname{expo}}=K_{\max }\left(1-e^{-\left(t \cdot t_{\text {tra }}\right)}\right)
$$

Where $K_{\max }$ is the function's final magnitude and $t_{\text {tra }}$ is the traveling time to reach a final destination. Figure 4 depicts these functions, with $t_{\text {tra }}=1 \mathrm{~s}$, and their first and second derivatives.
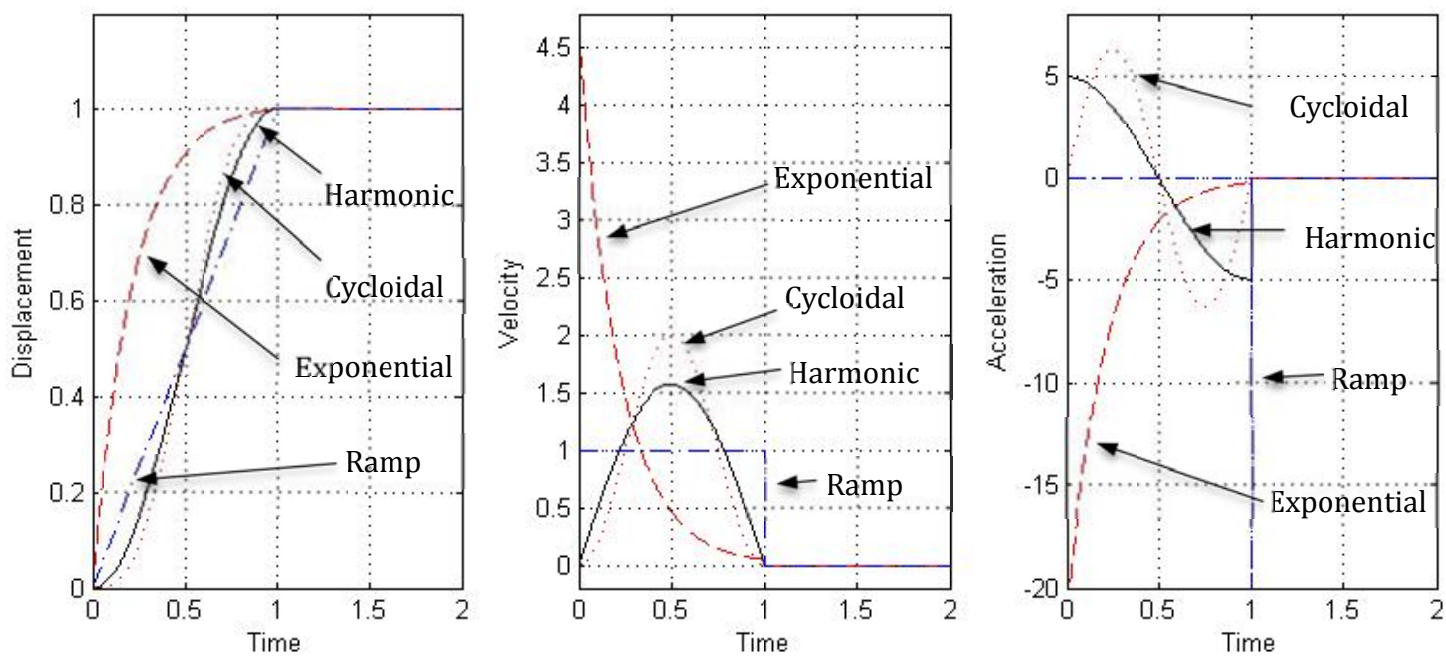

Figure 4 Motion characteristics of a shaped function.

\section{Simulated Feedback Control}

In our previous study [13], we proposed a new control method, which does not require an external displacement sensor for the feedback control of the CPA. This approach, called the 'simulated feedback control' (SFC) approach, uses a dynamic model of the actuator to obtain its displacement output for the feedback loop.

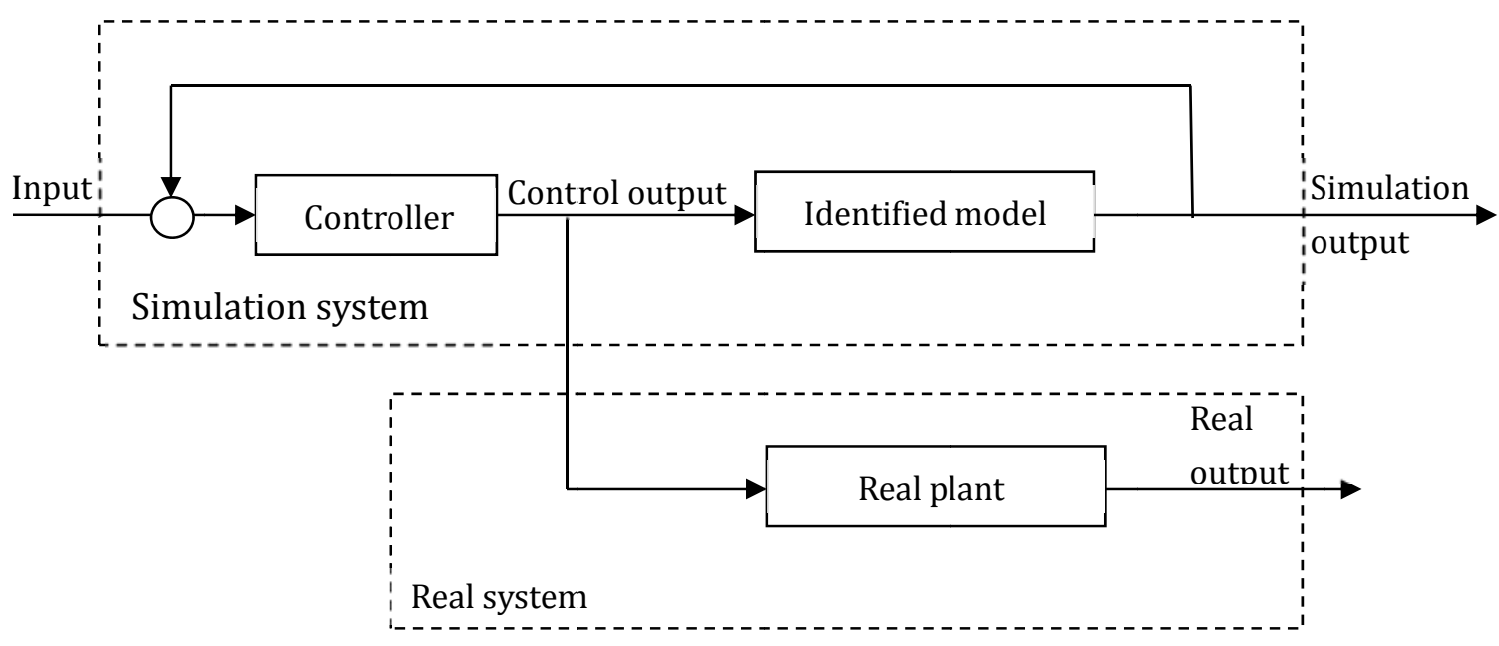

Figure 5 The schematic of the simulated feedback control siystem [13]

As shown in Figure 5, the feedback control is based on the identified model in the 
simulated environment. The controller's output is sent to the real plant (the CPA in our case) and the identified model. Theoretically, the simulation output and the real output should be the same because the "Identified model" and the "Real plant" are expected to be identical. In this way, we implement a closed-loop control algorithm without any physical feedback sensor. It must be noted that the control performance depends on the accuracy of the identified model. It is important to estimate the actuator model as accurately as possible.

\subsection{Controller Development}

\subsubsection{System Identification}

A range of models, from a second-order to a sixth-order, is considered to establish an accurate mathematical model. We have found that the second order model with a time delay fits the experimental data well. We use this model to eliminate the physical feedback sensor.

$G(s)=e^{-0.078 s} \frac{8.134 s+10.48}{s^{2}+5.194 s+1.728}$

The time delay can be represented by the following Pade approximation;

$$
G_{d}=\frac{s^{4}-256.4103 s^{3}+2.9586 \times 10^{4} s^{2}-1.7701 \times 10^{6}+4.5387 \times 10^{7}}{s^{4}+256.4103 s^{3}+2.9586 \times 10^{4} s^{2}+1.7701 \times 10^{6}+4.5387 \times 10^{7}}
$$

\subsubsection{Particle Swarm Optimization for Tuning PID Gains}

We employ a particle swarm optimization (PSO) to obtain the optimized PID gains for the controller in the SFC. PSO is an evolutionary computation technique to solve a nonlinear optimization problem. In this algorithm, a number of particles is placed in a multidimensional search space. Each of them flies in the space with a specific velocity. Every particle's velocity is dynamically adjusted according to its own flying experience and overall swarm flying experience. Eventually, the swarm, like a flock of birds capturing prey, will move close to the optimized point of the problem $[22,23]$.

For a PID controller, the control parameters, the proportional, integral and derivative gains, can be deemed as the three dimensions of one particle. A number of particles in the problem space search for their best position, which means the best gains. The criterion for the best position usually comes from a system response index in the time domain or the frequency domain. We employ a performance criterion $W(K)$ in our algorithm [24]. The performance criterion is given by

$$
W(K)=\left(1-e^{-\beta}\right) \cdot\left(M_{p}+E_{S S}\right)+e^{-\beta} \cdot\left(t_{s}-t_{r}\right)
$$

The overshoot $M_{p}$, rise time $t_{r}$, settling time $t_{s}$, and steady-state error $E_{s s}$ are incorporated into the criterion to obtain the gains satisfying this set of performance 
requirements. The numerical value of the parameter $\beta$ is changed to reflect the weighting of the performance requirements in the criterion.

We also consider the root mean square (RMS) of the error associated with the time response of the system under the identified gains. The set of PID gains resulting in the minimum RMS is used in the SFC. The RMS of the error is defined by:

$$
W^{\prime}(K)=R M S=\sqrt{\frac{1}{n} \sum_{i=1}^{n}\left(Y r_{i}-Y d_{i}\right)^{2}}
$$

where $n$ is the total number of data points, $Y_{r}$ is the real output, and $Y_{d}$ is the desired output.

It only takes 20 - 60 seconds to find the optimized PID gains for the controller. For the experimentally identified model, the PID gains of $k p=0.9404, k i=0.15687, k d=0$ are obtained.

\section{Dynamic Response under Open-loop Control}

We test the dynamic response under the open-loop control. We vary the travel time of each smooth input to command the actuator from an initial configuration to a final configuration. We have considered 5 travel times between 0.2 second and 1 second.

Figures 6-10 show the displacement response and the corresponding instantaneous power under a step input and 4 smooth functions with travel times of 0.2 second, 0.4 second, 0.6 second, 0.8 second and 1.00, second, respectively. Tables 1-5 show their corresponding time-domain performance characteristics. It must be noted that the travel time for a step input is zero.
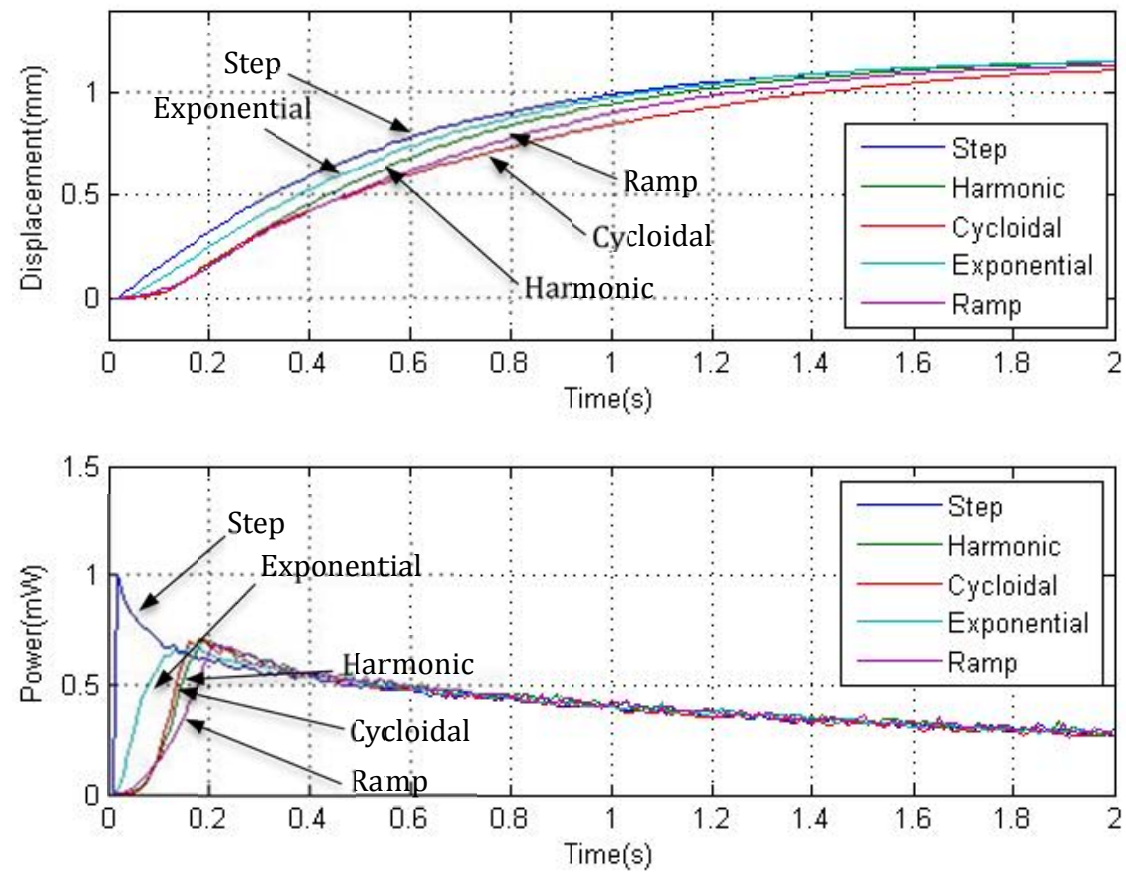
Figure 6 Displacement response (top plot) and power consumption (bottom plot) of the inputs with $0.2 \mathrm{~s}$ travel time under open-loop control for the first 2 seconds of the response.

Table 1. Displacement response characteristics of the inputs with $0.2 \mathrm{~s}$ travel time under the open-loop control

\begin{tabular}{ccccc}
\hline Input type & Overshoot (\%) & Rise time (s) & Settling time (s) & Energy applied (mJ) \\
\hline Step input & $7.47 \%$ & 0.9 & 8.37 & 0.85 \\
Harmonic & $6.8 \%$ & 0.95 & 7.67 & 0.81 \\
Cycloidal & $6.36 \%$ & 1.19 & 6.69 & 0.78 \\
Exponential & $7.46 \%$ & 0.91 & 6.49 & 0.83 \\
Ramp & $7.46 \%$ & 1.02 & 6.61 & 0.79 \\
\hline
\end{tabular}
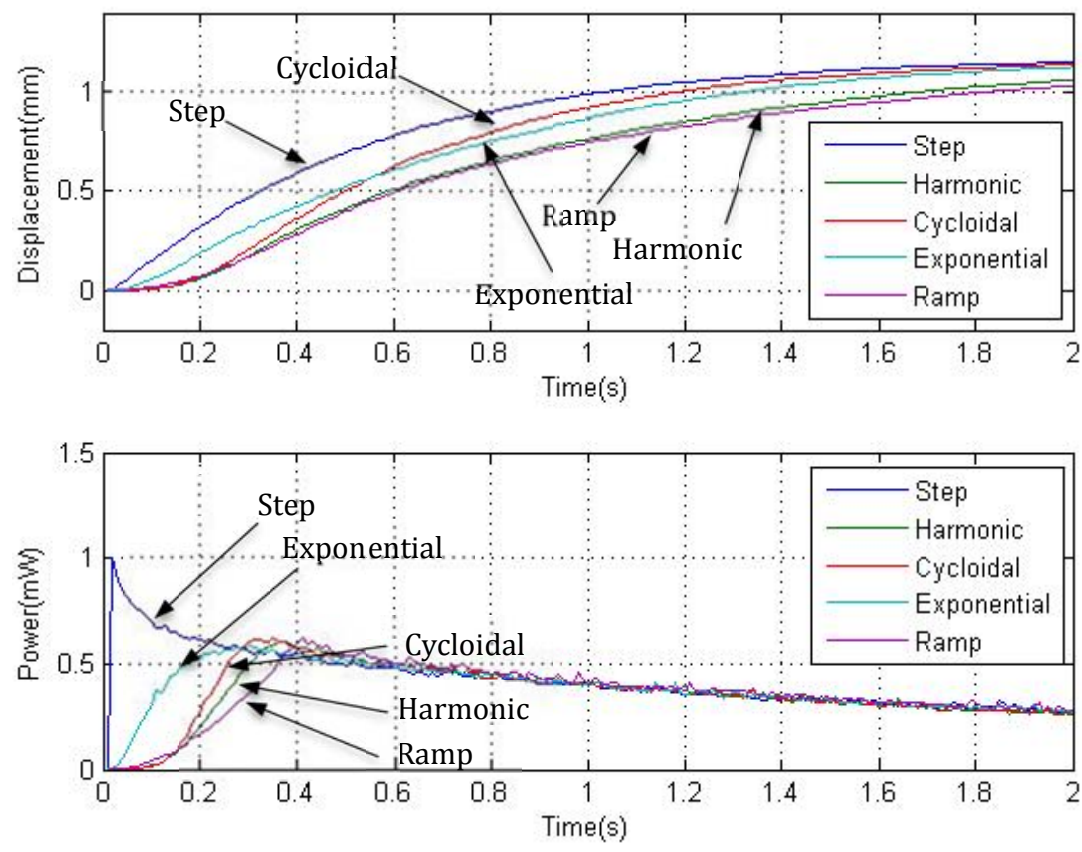

Figure 7 Displacement response (top plot) and power consumption (bottom plot) of the inputs with $0.4 \mathrm{~s}$ travel time under open-loop control

Table 2. Displacement Response Characteristics of the Inputs with $0.4 \mathrm{~s}$ Travel Time under open-loop control

\begin{tabular}{ccccc}
\hline Input type & Overshoot (\%) & Rise time (s) & Settling time (s) & Energy applied (mJ) \\
\hline Step input & $7.47 \%$ & 0.9 & 8.37 & 0.85 \\
Harmonic & $6.62 \%$ & 1.35 & 7.1 & 0.71 \\
Cycloidal & $9.13 \%$ & 0.85 & 8.31 & 0.73 \\
Exponential & $7.56 \%$ & 1.13 & 7.39 & 0.78 \\
Ramp & $5.91 \%$ & 1.47 & 6.59 & 0.72 \\
\hline
\end{tabular}



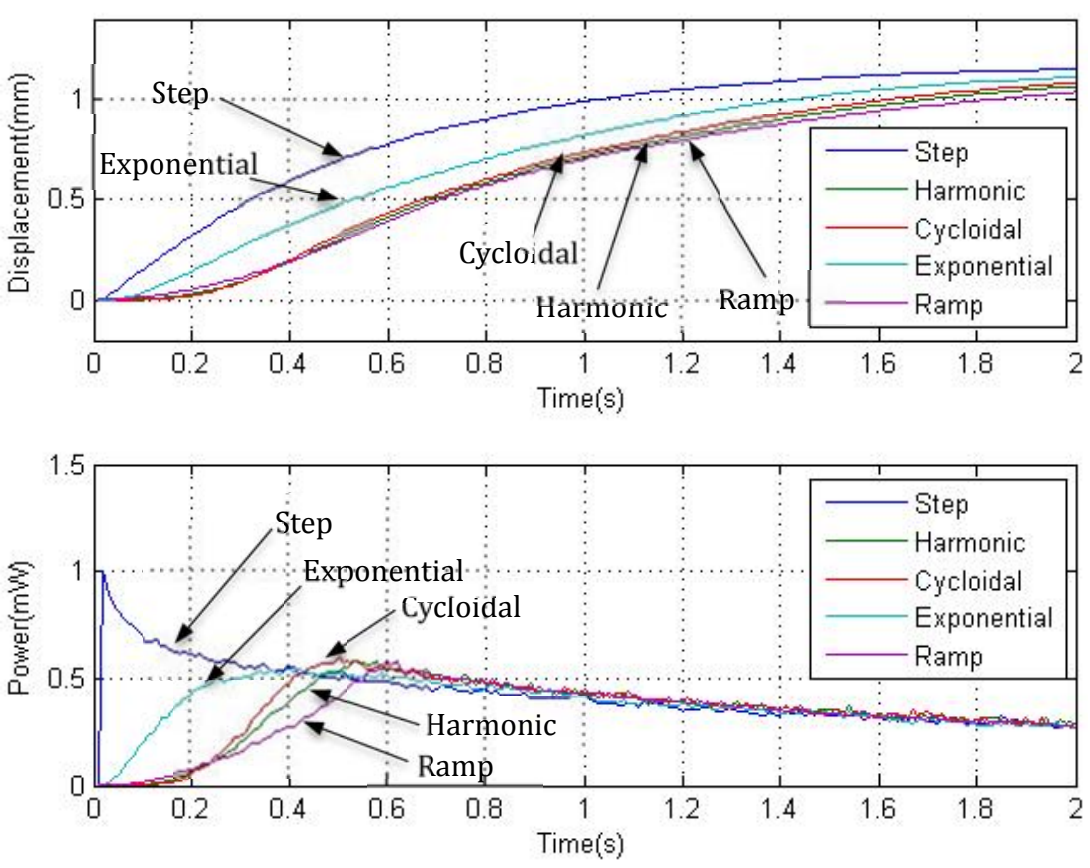

Figure 8 Displacement response (top plot) and power consumption (bottom plot) of the inputs with $0.6 \mathrm{~s}$ travel time under open-loop control

Table 3. Displacement Response Characteristics of the Inputs with $0.6 \mathrm{~s}$ Travel Time under open-loop control

\begin{tabular}{ccccc}
\hline Input type & Overshoot (\%) & Rise time (s) & Settling time (s) & Energy applied (mJ) \\
\hline Step input & $7.47 \%$ & 0.9 & 8.37 & 0.85 \\
Harmonic & $7.23 \%$ & 1.29 & 7.51 & 0.69 \\
Cycloidal & $7.69 \%$ & 1.21 & 7.67 & 0.70 \\
Exponential & $7.87 \%$ & 1.19 & 7.78 & 0.76 \\
Ramp & $6.85 \%$ & 1.45 & 7.39 & 0.66 \\
\hline
\end{tabular}



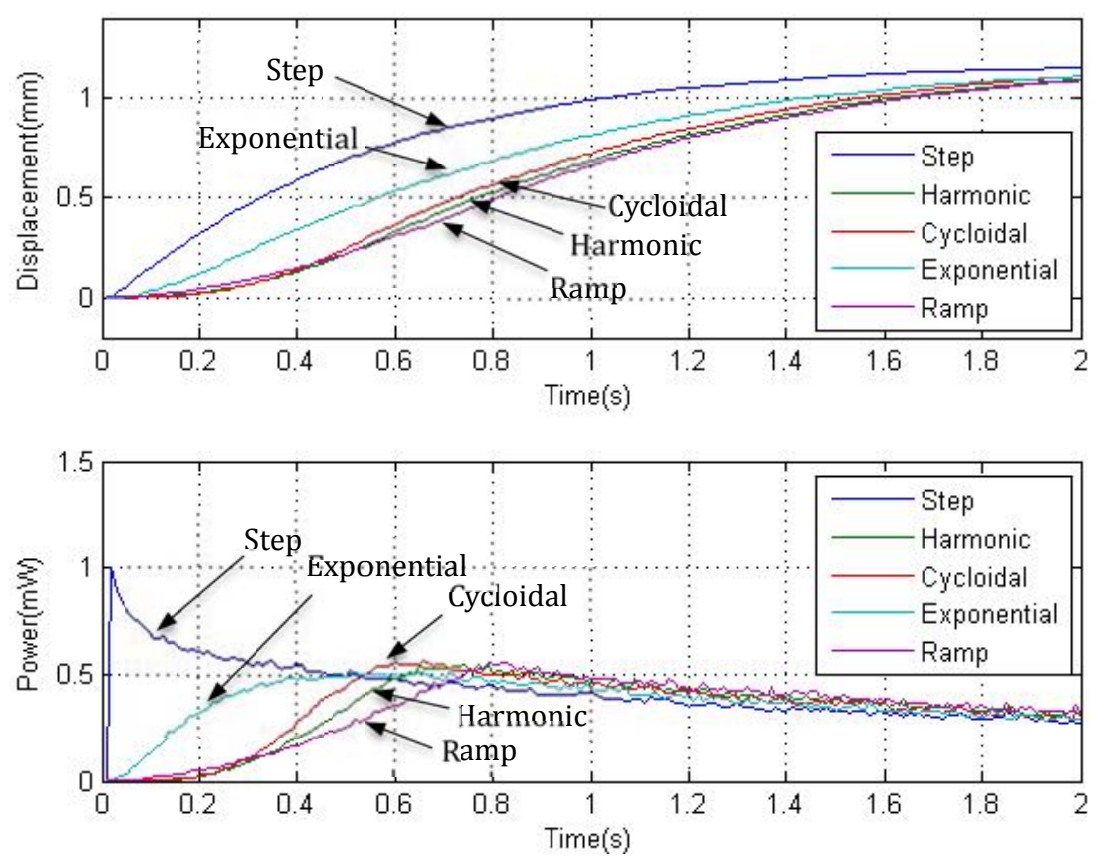

Figure 9 Displacement response (top plot) and power consumption (bottom plot) of the inputs with 0.8 s travel time under open-loop control.

Table 4. Displacement Response Characteristics of the Inputs with $0.8 \mathrm{~s}$ Travel Time under open-loop control

\begin{tabular}{ccccc}
\hline Input type & Overshoot (\%) & Rise time (s) & Settling time (s) & Energy applied (mJ) \\
\hline Step input & $7.47 \%$ & 0.9 & 8.37 & 0.85 \\
Harmonic & $7.62 \%$ & 1.21 & 7.41 & 0.67 \\
Cycloidal & $7.43 \%$ & 1.16 & 7.29 & 0.68 \\
Exponential & $7.46 \%$ & 1.19 & 6.95 & 0.75 \\
Ramp & $7.97 \%$ & 1.26 & 7.87 & 0.67 \\
\hline
\end{tabular}



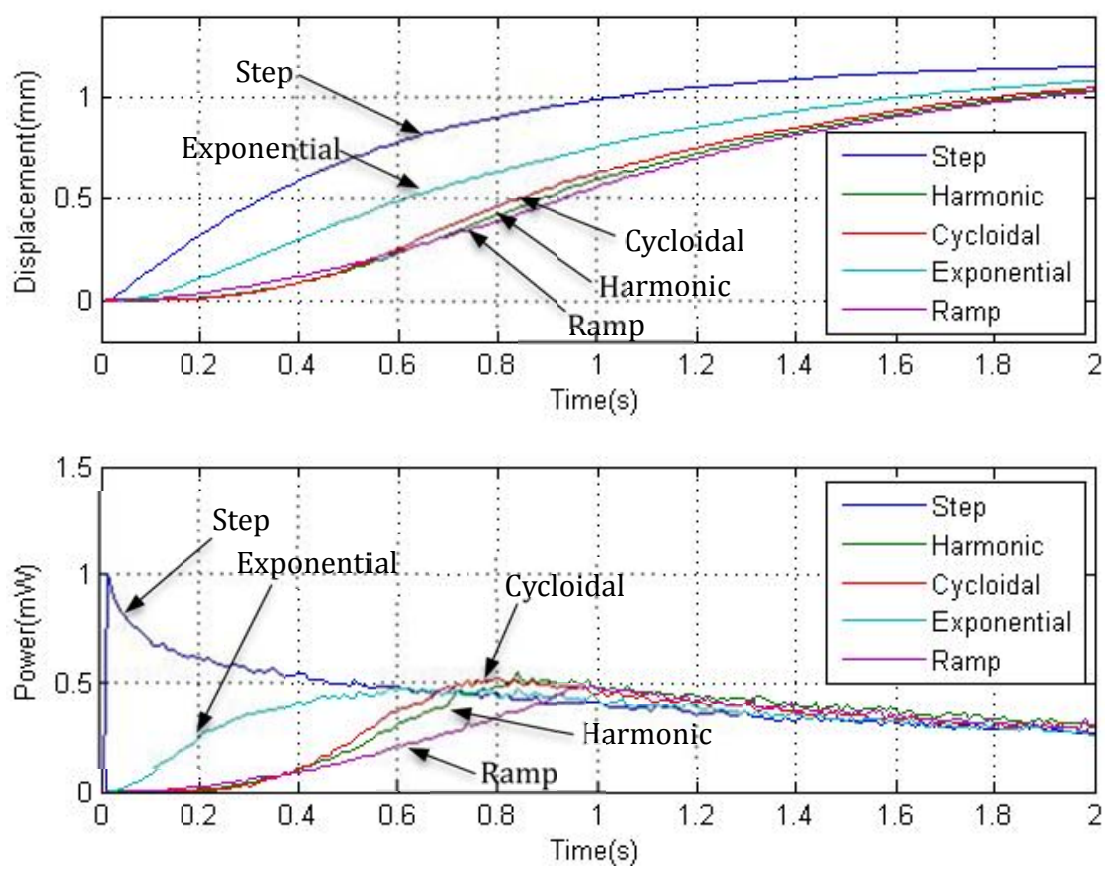

Figure 10 Displacement response (top plot) and power consumption (bottom plot) of the inputs with 1.0 s travel time under open-loop control

Table 5. Displacement Response Characteristics of the Inputs with 1.0s Travel Time under open-loop control

\begin{tabular}{ccccc}
\hline Input type & Overshoot (\%) & Rise time (s) & Settling time (s) & Energy applied (mJ) \\
\hline Step input & $7.47 \%$ & 0.9 & 8.37 & 0.85 \\
Harmonic & $7.01 \%$ & 1.34 & 7.92 & 0.62 \\
Cycloidal & $7.50 \%$ & 1.31 & 8.2 & 0.61 \\
Exponential & $7.30 \%$ & 1.34 & 7.79 & 0.69 \\
Ramp & $6.78 \%$ & 1.44 & 7.63 & 0.55 \\
\hline
\end{tabular}

These results show that the relatively smooth inputs consume less electrical energy under open-loop control. Though the rise time of the responses under the functional smooth inputs is longer compared to a step input, the settling times under these inputs are even shorter than that of the step input. The smooth inputs show a slightly better dynamic performance with significantly less energy consumption when compared to the step input.

\section{Dynamic Response under Simulated Feedback Control}

As presented in Section 4, a smooth input consumes a relatively lower power with a small price of slightly impaired transient response characteristics as listed in Tables 1-5. Because of the advantage of the closed-loop control strategy; we postulate that this impairment will be minimized under the SFC approach, still consuming less power under the smooth inputs. To verify this hypothesis, we experimentally evaluated the performance of the smooth inputs with different travel times, from 0.2 second to 1.0 second. 


\subsection{Actuator Response with 0.2s Travel under Simulated Feedback Control}

Figure 11 and Table 6 depict the displacement, instantaneous power, control voltage and the time-domain performance characteristics of the response under 5 functions with a travel time of 0.2 second.
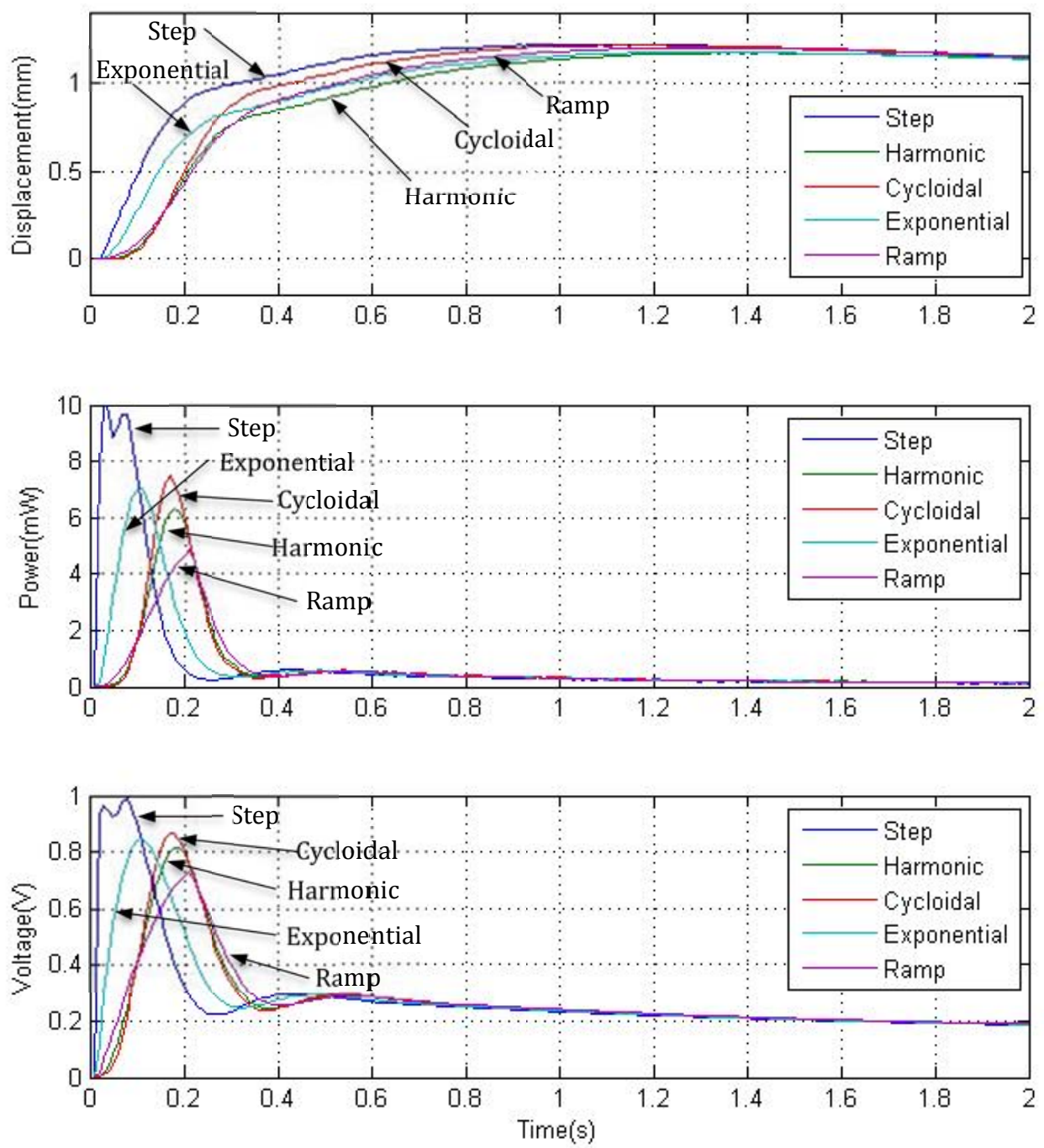

Figure 11 Displacement response (1st), power consumption (2nd) and control voltage (3rd) applied to the actuator under smooth inputs with $0.2 \mathrm{~s}$ travel time using the SFC approach.

Table 6. Displacement Response Characteristics of the Inputs with $0.2 \mathrm{~s}$ Travel Time.

\begin{tabular}{cccccc}
\hline Input type & $\begin{array}{c}\text { Overshoot } \\
\mathbf{( \% )}\end{array}$ & Rise time (s) & Settling time (s) & RMS & $\begin{array}{c}\text { Energy } \\
\text { applied (mJ) }\end{array}$ \\
\hline Step input & $21.86 \%$ & 0.18 & 3.29 & 0.081 & 1.60 \\
Harmonic & $17.07 \%$ & 0.41 & 3.44 & 0.071 & 1.31 \\
Cycloidal & $21.27 \%$ & 0.21 & 3.26 & 0.075 & 1.37 \\
\hline
\end{tabular}




\begin{tabular}{cccccc}
\hline Exponential & $17.55 \%$ & 0.37 & 3.26 & 0.073 & 1.39 \\
Ramp & $19.97 \%$ & 0.32 & 3.5 & 0.072 & 1.19 \\
\hline
\end{tabular}

\subsection{Actuator Response with 0.4s Travel under Simulated Feedback Control}

Figure 12 and Table 7 depict the displacement, instantaneous power, control voltage and the time-domain performance characteristics of the response under 5 functions with a travel time of 0.4 second.
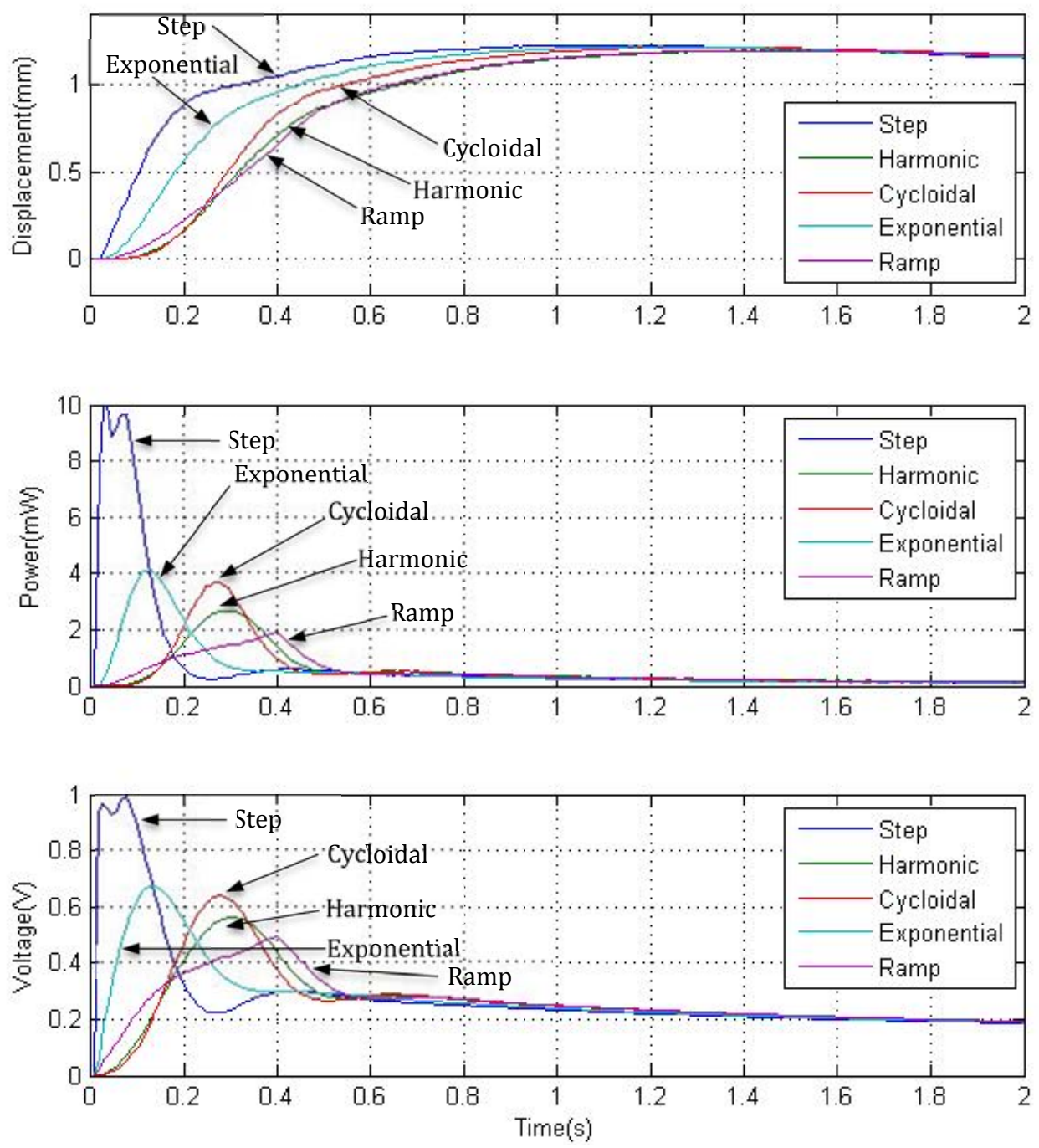

Figure 12 Displacement response (1st), power consumption (2nd) and control voltage (3rd) applied to the actuator under the smooth inputs with $0.4 \mathrm{~s}$ travel time under SFC approach.

Table 7. Displacement Response Characteristics of the Inputs with $0.4 \mathrm{~s}$ Travel Time.

\begin{tabular}{lccccc}
\hline Input type & $\begin{array}{c}\text { Overshoot } \\
\text { (\%) }\end{array}$ & $\begin{array}{c}\text { Rise time } \\
\text { (s) }\end{array}$ & $\begin{array}{c}\text { Settling time } \\
\text { (s) }\end{array}$ & RMS & $\begin{array}{c}\text { Energy } \\
\text { applied (m)) }\end{array}$ \\
\hline Step input & $21.86 \%$ & 0.18 & 3.29 & 0.081 & 1.60 \\
\hline
\end{tabular}




\begin{tabular}{cccccc}
\hline Harmonic & $18.79 \%$ & 0.38 & 3.47 & 0.069 & 1.05 \\
Cycloidal & $20.91 \%$ & 0.28 & 3.46 & 0.072 & 1.11 \\
Exponential & $21.31 \%$ & 0.28 & 3.38 & 0.073 & 1.08 \\
Ramp & $19.23 \%$ & 0.41 & 3.61 & 0.068 & 0.93 \\
\hline
\end{tabular}

\subsection{Actuator Response with 0.6s Travel under Simulated Feedback Control}

Figure 13 and Table 8 depict the displacement, instantaneous power, control voltage and the time-domain performance characteristics of the response under 5 functions with a travel time of 0.6 second.


Figure 13 Displacement response (1st), power consumption (2nd) and control voltage (3rd) applied to the actuator under the smooth inputs with $0.6 \mathrm{~s}$ travel time using the SFC approach.

Table 8. Displacement Response Characteristics of the Inputs with $0.6 \mathrm{~s}$ Travel Time. 


\begin{tabular}{cccccc}
\hline Input type & $\begin{array}{c}\text { Overshoot } \\
\text { (\%) }\end{array}$ & $\begin{array}{c}\text { Rise time } \\
\text { (s) }\end{array}$ & $\begin{array}{c}\text { Settling time } \\
\text { (s) }\end{array}$ & RMS & $\begin{array}{c}\text { Energy } \\
\text { applied (m) }\end{array}$ \\
\hline Step input & $21.86 \%$ & 0.18 & 3.29 & 0.081 & 1.60 \\
\hline Harmonic & $20.37 \%$ & 0.39 & 3.57 & 0.067 & 0.91 \\
Cycloidal & $20.09 \%$ & 0.33 & 3.47 & 0.065 & 0.91 \\
\hline Exponential & $19,79 \%$ & 0.37 & 3.42 & 0.069 & 1.08 \\
\hline Ramp & $17.66 \%$ & 0.53 & 3.55 & 0.065 & 0.89 \\
\hline
\end{tabular}

\subsection{Actuator Response with 0.8s Travel under Simulated Feedback Control}

Figure 14 and Table 9 depict the displacement, instantaneous power, control voltage and the time-domain performance characteristics of the response under 5 functions with a travel time of 0.8 second.


Figure 14 Displacement response (1st), power consumption (2nd) and control voltage (3rd) applied to the actuator under the smooth inputs with $0.8 \mathrm{~s}$ travel time under SFC approach. 
Table 9. Displacement Response Characteristics of the Inputs with $0.8 \mathrm{~s}$ Travel Time.

\begin{tabular}{cccccc}
\hline Input type & $\begin{array}{c}\text { Overshoot } \\
\text { (\%) }\end{array}$ & $\begin{array}{c}\text { Rise time } \\
\text { (s) }\end{array}$ & $\begin{array}{c}\text { Settling time } \\
\text { (s) }\end{array}$ & RMS & $\begin{array}{c}\text { Energy } \\
\text { applied (m) }\end{array}$ \\
\hline Step input & $21.86 \%$ & 0.18 & 3.29 & 0.081 & 1.60 \\
\hline Harmonic & $18.46 \%$ & 0.53 & 3.81 & 0.064 & 0.84 \\
Cycloidal & $19.30 \%$ & 0.41 & 3.55 & 0.063 & 0.93 \\
Exponential & $18.67 \%$ & 0.46 & 3.6 & 0.065 & 1.02 \\
\hline Ramp & $18.31 \%$ & 0.61 & 3.75 & 0.060 & 1.76 \\
\hline
\end{tabular}

\subsection{Actuator Response with 1.0s Travel under Simulated Feedback Control}

Figure 15 and Table 10 depict the displacement, instantaneous power, control voltage and the time-domain performance characteristics of the response under 5 functions with a travel time of 1.0 second.
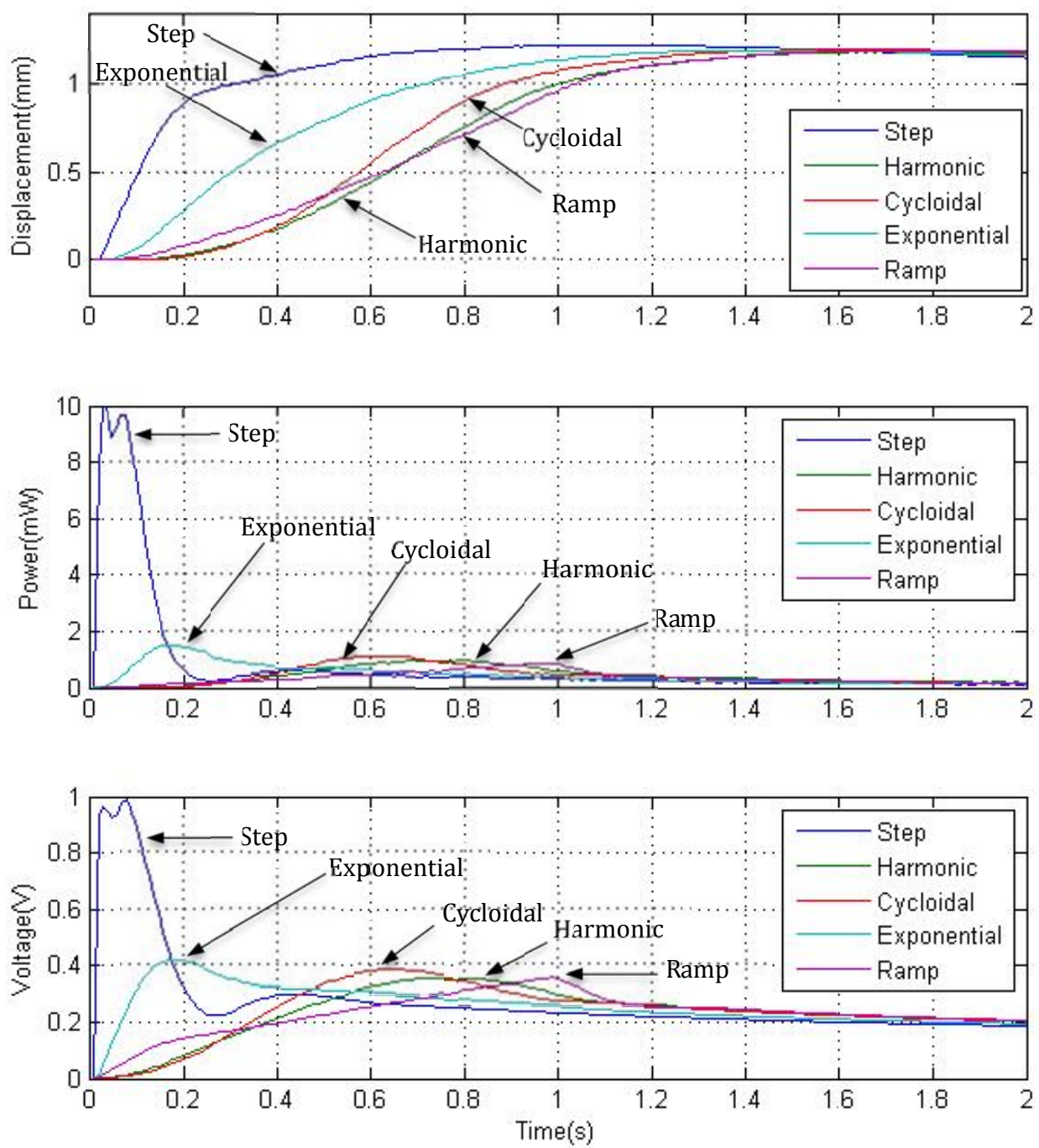
Figure 15 Displacement response (1st), power consumption (2nd) and control voltage (3rd) applied to the actuator under the smooth inputs with $1.0 \mathrm{~s}$ travel time under SFC approach.

Table 10. Displacement Response Characteristics of the Inputs with $1.0 \mathrm{~s}$ Travel Time.

\begin{tabular}{cccccc}
\hline Input type & $\begin{array}{c}\text { Overshoot } \\
\text { (\%) }\end{array}$ & $\begin{array}{c}\text { Rise time } \\
\text { (s) }\end{array}$ & $\begin{array}{c}\text { Settling time } \\
\text { (s) }\end{array}$ & RMS & $\begin{array}{c}\text { Energy } \\
\text { applied (mJ) }\end{array}$ \\
\hline Step input & $21.86 \%$ & 0.18 & 3.29 & 0.081 & 1.60 \\
Harmonic & $18.23 \%$ & 0.6 & 3.99 & 0.063 & 0.82 \\
Cycloidal & $19.53 \%$ & 0.48 & 3.8 & 0.061 & 0.80 \\
Exponential & $18.95 \%$ & 0.49 & 3.76 & 0.067 & 0.89 \\
Ramp & $18.81 \%$ & 0.74 & 3.97 & 0.057 & 0.68 \\
\hline
\end{tabular}

\subsection{Summary}

With reference to the response characteristics presented in this section, the smooth inputs enable the actuator to provide a better system performance. For example, compared to the step input, the cycloidal input with $0.8 \mathrm{~s}$ travel time improves the steady state error by $71.4 \%$, the error RMS by $22.2 \%$, overshoot by $11.7 \%$ and consumes $41.9 \%$ less energy.

The harmonic input shows the most favorable performance for a short travel time. The ramp input provides the most favorable performance for a long travel time. Without increasing the travel time, we can choose a cycloidal or exponential input to obtain a fast response. All smooth inputs require less energy consumption than a step input. The ramp input consumes the smallest energy to command the actuator from one configuration to the other.

\section{Steady State Response}

Inputs with different magnitudes, from $0.02 \mathrm{~V}$ to $0.22 \mathrm{~V}$, were applied to a conducting polymer actuator to evaluate its steady-state response. The steady-state displacement responses are shown in Figure 16-Figure 18. 

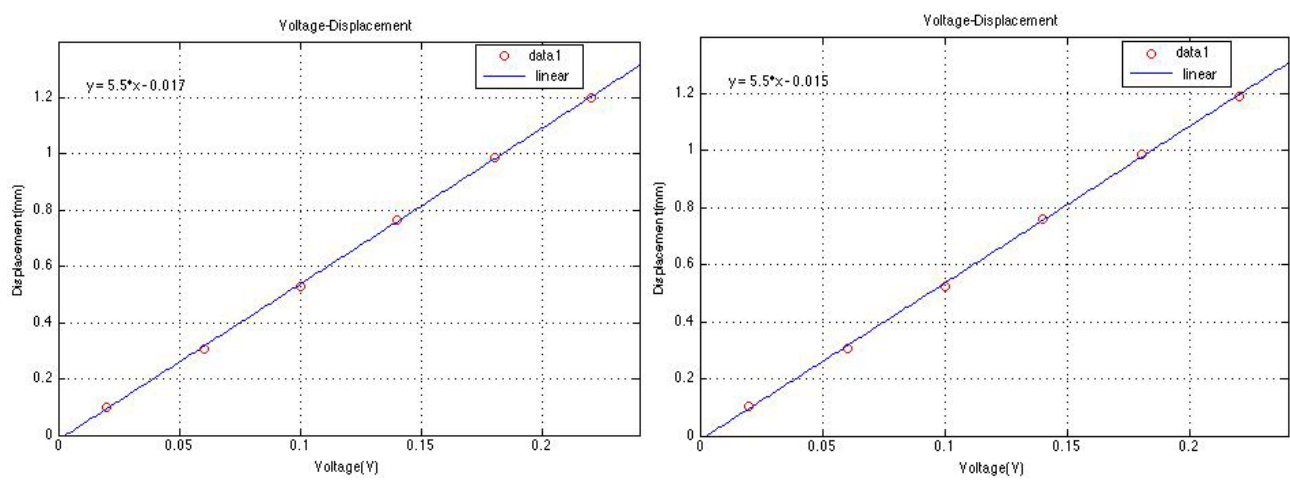

Figure 16 The steady-state displacements under step inputs (left side) and harmonic inputs (right side).
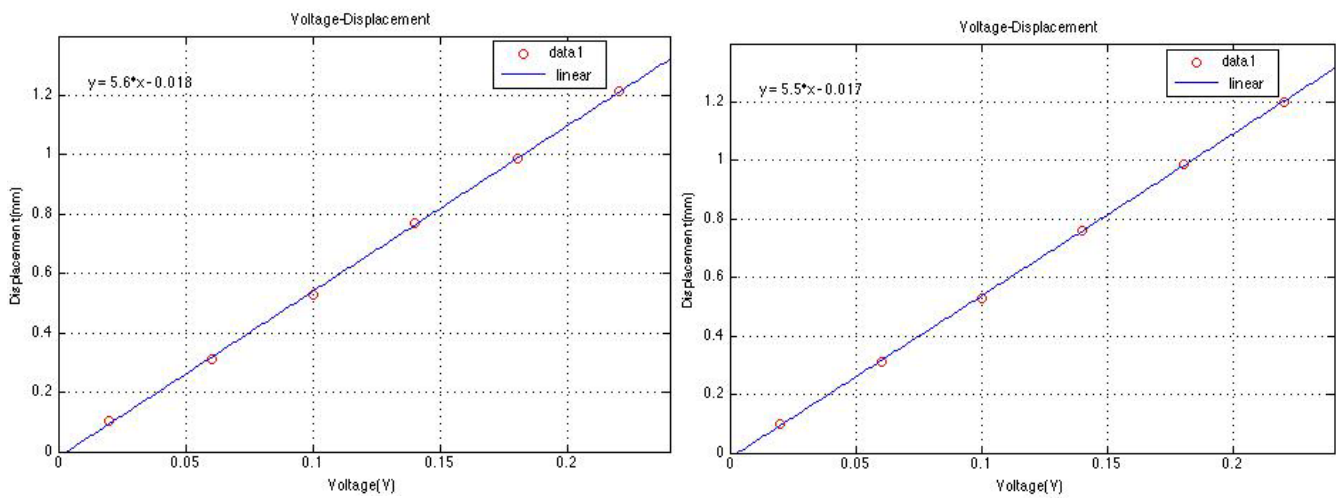

Figure 17 The steady-state displacements under cycloidal input (left side) and exponential inputs (right side).

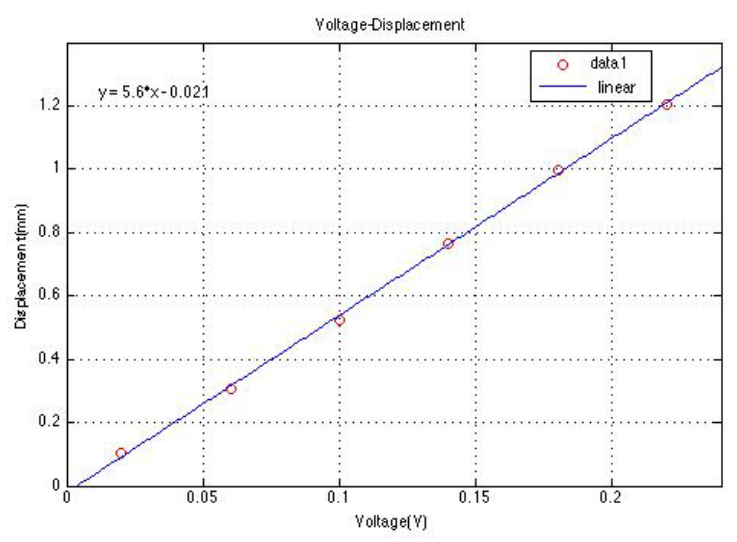

Figure 18 The steady-state displacements under ramp inputs.

The relationship between the steady-state displacement output and the magnitude of the input voltage can be represented by a linear function, as shown in Table 11. These results suggest that there is no obvious difference between the linear functions. Figure 19 also shows that these functions almost overlap, which means the type or smoothness of the input does not affect the actuator's steady-state response.

Table 11. The fitted function of the magnitude-displacement relationship

\begin{tabular}{|c|c|}
\hline Input type & Fitted Function \\
\hline Step Input & $\mathrm{y}=5.5475 * \mathrm{x}-0.017355$ \\
\hline
\end{tabular}




\begin{tabular}{|c|c|}
\hline Harmonic Input & $\mathrm{y}=5.5049 * \mathrm{x}-0.014855$ \\
\hline Cycloidal Input & $\mathrm{y}=5.5835 * \mathrm{x}-0.018371$ \\
\hline Exponential Input & $\mathrm{y}=5.5457 * \mathrm{x}-0.017403$ \\
\hline Ramp Input & $\mathrm{y}=5.5932 * \mathrm{x}-0.021264$ \\
\hline
\end{tabular}

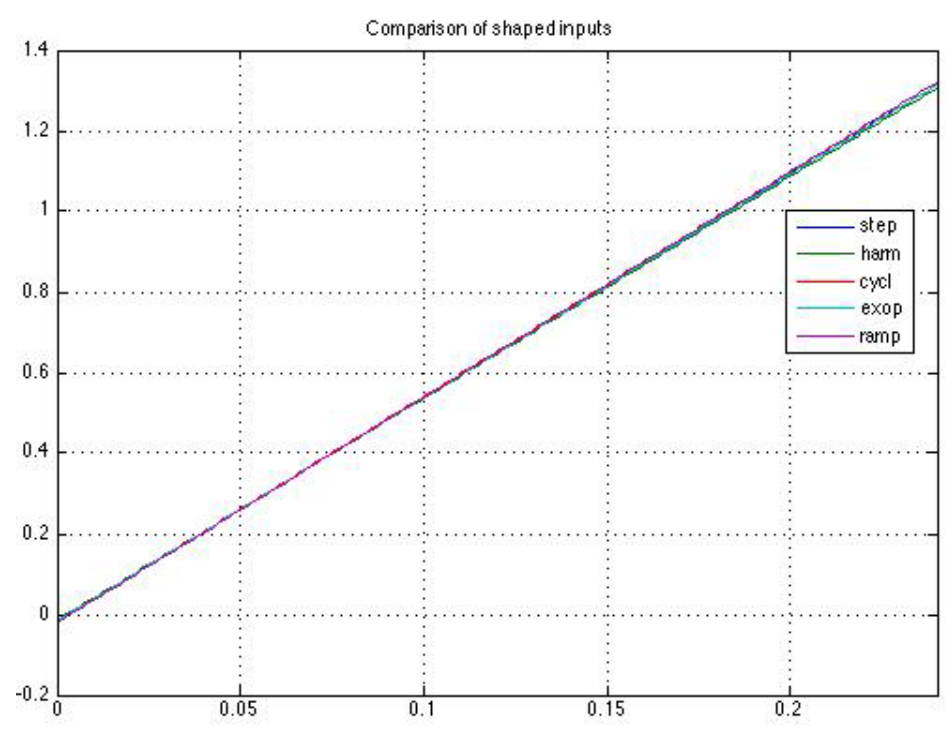

Figure 19 The comparison of the fitted functions of the magnitude-displacement relationships of step and shaped Inputs.

\section{Discussions and Conclusion}

This study has investigated the effect of the input signal on the dynamic response characteristics and power consumption of smart actuators typified by trilayer electroactive actuators. Under open-loop control, the smooth inputs of ramp, cycloid, harmonic and exponential functions require less energy to bring the actuator to the same steady-state configuration, and make the actuator show a slightly better performance, compared to a step input. Consuming less electric energy or power has a direct effect on enhancing the operational life of these actuators. Under a simulated feedback control approach (SFC), as a result of the controlled input voltage applied on the conducting polymer actuators, these favorable characteristics are even more obvious. Energy consumed, overshoot and RMS of the tracking error are also significantly improved.

The favorable performance under relatively smooth inputs can be explained by the fact that enough time is given to the ionic species (i.e anions and cations) to move in and out of the PPy layers, rather than congesting the interface between the PPy layers and the PVDF layer under a sharp and instantaneous energy input such as a step input. Under a step-like input, the anions are driven to move into the porosities in the oxidized PPy layer, but there is a limit to the number of ions that can be passed through the porosities inside the PPy layer to generate the volume expansion. As a result, a smooth input, will make the actuator show a more satisfactory overall performance and consume less energy to reach the same steady-state configuration. 
We have also found that the steady state displacement is almost the same under all the smooth inputs and step input, which means the smooth inputs enhance the transient response of the conducting polymer actuators but do not affect their steady-state response. Future work will focus on (i) quantifying how much improvement can be made in the operational life of these actuators, and (ii) shaping multi-piece input functions (e.g. ramp + cycloidal, ramp + harmonic, and similar) resulting in even better performance characteristics.

\section{Acknowledgements:}

This work was supported by the ARC Centre of Excellence for Electromaterials Science (Grant No. CE0561616). The authors wish to gratefully acknowledge the help of Dr. Madeleine Strong Cincotta in the final language editing of this paper.

\section{References}

[1] K. Kiyohara, T. Sugino, and K. Asaka "Molecular mechanism of ionic electroactive polymer actuators", Smart Materials and Structures, vol. 20, no.12, Nov. 2011.

[2] E. Smela, "Conjugated polymer actuators for biomedical applications," Advanced Materials, vol. 15, pp. 481-494, 2003.

[3] G.M. Spinks, G. Alici, S. McGovern, B. Xi and G.G. Wallace, "Fundamental Mechanisms of Actuation in Conducting Polymers", A Chapter in Biomedical Applications of Electroactive Polymer Actuators edited by F. Carpi and E. Smela, John Wiley \& Sons, Ltd., Chichester, UK, pp. 195-228, 2009.

[4] J.D.W. Madden, “Conducting polymer Actuators," Ph.D. dissertation, Massachusetts Inst. Technol., Cambridge, MA, USA , 2000.

[5] R. H. Baughman, L. W. Shacklette, R. L. Elsenbaumer, E. Plichta, C. Becht, "Conjugated Polymeric Materials: Opporunities in Electronics, Optoelectronics, and Molecular Electronics" (Eds: J. L. BrØdas, R. R. Chance), Vol. 182, Kluwer, Dordrecht, The Netherlands 1990.

[6] G. G. Wallace, G. M. Spinks, P. R. Teasdale, "Conductive Electroactive Polymers: Intelligent Materials Systems”, Technomic, Lancaster, UK 1997.

[7] S. W. John, G. Alici, and C. D. Cook, "Inversion-Based Feedforward Control of Polypyrrole Trilayer Bender Actuators," IEEE/ASME Transactions on Mechatronics, vol. 15, pp. 149-156, 2010.

[8] Anwar, M. M., Saleh, T., Madden, J. D. W. and Takahata, K. (2014), Micropatterning Polypyrrole Conducting Polymer by Pulsed Electrical Discharge. Macromolecular Materials and Engineering, 299: 198-207.

[9] Q. Yao, G. Alici, and G. M. Spinks, "Feedback control of tri-layer polymer actuators to improve their positioning ability and speed of response," Sensors and Actuators A-Physical, vol. 144, pp. 176-184, 2008.

[10] Shoa, T., Dan Sik Yoo, Walus, K. \& Madden, J.D.W. 2011, "A Dynamic Electromechanical Model for Electrochemically Driven Conducting Polymer Actuators", IEEE/ASME Transactions on Mechatronics, vol. 16, no. 1, pp. 42-49 
[11] F. Yang, X. Tan, and G. Alici, "Robust Adaptive Control of Conjugated Polymer Actuators," IEEE Transactions on Control System Technology, vol. 16, pp. 600-612, 2008.

[12] X. Wang, G. Alici, and C. H. Nguyen, "Adaptive sliding mode control of tri-layer conjugated polymer actuators," Smart Materials and Structures, vol. 22, pp. 1-8, 2013.

[13] X. Xiang, R. Mutlu, G. Alici, and W. Li, "Control of conducting polymer actuators without physical feedback: simulated feedback control approach with particle swarm optimization", Journal of Smart Materials and Structure, vol. 23, no. 3, 2014.

[14] A. D. Price, and H. E. Naguib, "A unified multiphysics finite element model of the polypyrrole trilayer actuation mechanism", J. of Intelligent Material Systems and Structures, vol. 24, no. 5, pp. 548-558, 2013.

[15] C. H. Nguyen, G. Alici, and G. G. Wallace, "Modelling trilayer conjugated polymer actuators for their sensorless position control," Sensors and Actuators A-Physical, vol. 185, pp. 82-91, 2012.

[16] John S W, Alici G and Cook C D 2009 "Towards the position control of conducting polymer trilayer bending actuators with integrated feedback" sensor IEEE/ASME Int. Conf. on Advance Intelligent Mechatronics, 14-17 July 2009, Singapore pp 65-70

[17] Alici G, Devaud V, Renaud P and Spinks G 2009 "Conducting polymer microactuators operating in air" J. Micromech. Microeng. 19025017

[18] S. Kapucu, G. Alici, and S. Baysec, "Residual Swing / Vibration Reduction Using a Hybrid Input Shaping Method", Mechanism and Machine Theory, vol 36, no.3, pp.311 - 326, 2001

[19] G. Alici, S. Kapucu and S. Baysec, "On Preshaped Reference Inputs To Reduce Swing of Suspended Objects Transported With Robot Manipulators", Mechatronics: An International Journal, 10:6, pp. 609 - 626, 2000.

[20] G. Alici, S. Kapucu and S. Baysec, "Swing-free Transportation of Suspended Objects with Robot Manipulators", Robotica, volume 17, pp.513 -521, 1999.

[21] M. F. Daqaq, C. K. Reddy and A. H. Nayfeh, "Input-shaping control of nonlinear MEMS" Nonlinear Dynamics, Vol. 54, no. 1-2, pp. 167-179. 2008.

[22] J. Kennedy and R. Eberhart, "Particle swarm optimization " in IEEE International Conference on Neural Networks, Perth, WA, 1995, pp. 1942-1948.

[23] R. Poli, J. Kennedy, and T. Blackwell, "Particle swarm optimization: An overview," Swarm Intelligence, vol. 1, pp. 33-57, 2007.

[24] Z.-L. Gaing, "A particle swarm optimization approach for optimum design of PID controller in AVR system," IEEE Transactions on Energy Conversion, vol. 19, pp. 384-391, 2004. 